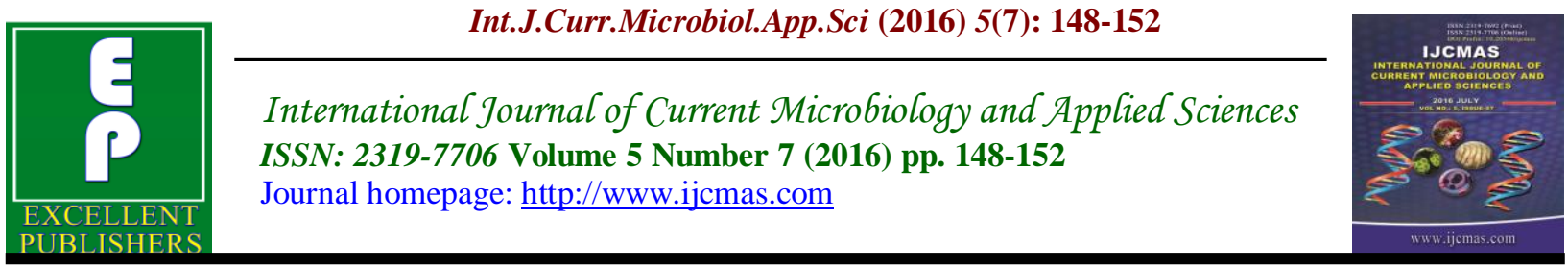

Case Study

http://dx.doi.org/10.20546/ijcmas.2016.507.014

\title{
Lymphangioleiomyomatosis in a 55 Year Old Female -A Rare Case Report
}

\author{
Krishna Bharadwaj Reddy*, V. Gopala Krishniaiah and Y. Murali
}

Department of pulmonary medicine Bhaskar Medical college and

General hospital Moinabad, RR district, Telangana state, India

*Corresponding author

A B S T R A C T

Keywords

Lymphangioleiomyomatosis (LAM),

Angiomyolipomas

[AML],

Tuberous sclerosis complex (TSC)

DLCO -Diffusion

capacity of lung.

Article Info

Accepted:

08 June 2016

Available Online:

10 July 2016
Lymphangioleiomyomatosis (LAM) is a rare disorder resulting from proliferation in the lung, kidney, and axial lymphatics of abnormal smooth muscle-like cells (LAM cells) that exhibit features of neoplasia and neural crest origin. Cystic destruction of the lung with progressive pulmonary dysfunction and the presence of abdominal tumors.LAM can occur with increased frequency in patients with tuberous sclerosis complex (TSC), an autosomal dominant disorder due, in part, to mutations in the TSC1 or TSC2 gene. Here in the study present a 55 year old female with shortness of breath and reduced exercise capacity after complete investigation diagnosed as Lymphangioleiomyomatosis.Though there is availability of excellent imaging methods, histological examination are the gold standard tools for the final diagnosis.

\section{Introduction}

Lymphangioleiomyomatosis (LAM) is a rare disorder resulting from proliferation in the lung, kidney, and axial lymphatics of abnormal smooth muscle-like cells (LAM cells) that exhibit features of neoplasia and neural crest origin (Johnson et al., 1999). Cystic destruction of the lung with progressive pulmonary dysfunction and the presence of abdominal tumors (eg, angiomyolipomas [AML], lymphangioleiomyomas) characterize the disease. LAM typically occurs in premenopausal women, suggesting the involvement of female hormones in disease pathogenesis. LAM can occur with increased frequency in patients with tuberous sclerosis complex (TSC), an autosomal dominant disorder due, in part, to mutations in the TSC 1 or TSC 2 gene. Here we present a 55 year old female with shortness of breath and reduced exercise capacity, cough, reversible airway obstruction responding to bronchodilators, DLCO showing deduced gas diffusion.FEV1/FVC ratio is decreased (NHLBI, 1999). 


\section{Case Report}

A 55 year old female presented with shortness of breath since 1 year which is insidious in onset, started as grade 1 and slowly progressed to grade 2 over a period of one year. Occasional cough, history of recurrent lower respiratory tract infections, no expectoration, no history of chest pain, no history of hemoptysis on examination patient is afebrile, pulse $88 / \mathrm{min}$, respiratory rate $18 / \mathrm{min}$, no clubbing, no lymphadenopathy, no edema, no cyanosis, no jaundice. Bilateral crackles are heard in the late inspiratory phase, no absent breath sounds, no shift of mediastinum, bilateral respiratory movements are equal.

Chest radiograph showed bilateral basal reticular pattern high-resolution CT scan findings include the following: Diffuse thinwalled cysts - The defining appearance in LAM, Ground-glass opacities, Multifocalmultinodular pneumocyte hyperplasia (MMPH), Abdominal imaging Angiomyolipoma (AML) - Benign tumors (kidney, liver, spleen) containing smooth muscle, abnormal blood vessels, and mature adipose tissue Retroperitoneal and mediastinal lymphangioleiomyomas bone densitometry, showed osteoporosis Pulmonary function testing, decreased diffusing capacity for carbon monoxide markedly reduced.

On spirometry, airflow obstruction with reversibility with bronchodilator is seen. CT guided Transthorasic needle biopsy done which showed In histological sections of the lung, the following are observed Proliferation of lymphangioleiomyomatosis (LAM) cells (spindle-shaped cells with small nuclei, larger epithelioid cells with clear cytoplasm and round nuclei) having a smooth muscle cell phenotype. Loss of alveoli with cyst formation. Aggregates of LAM cells abutting cystic spaces Distal airway narrowing, thickened arterial walls with venous occlusion, and hemosiderosis.

\section{Results and Discussion}

Proliferation of lymphangioleiomyomatosis (LAM) cells may obstruct bronchioles, leading to airflow obstruction, air trapping, formation of bullae, and pneumothoraces (Taylor et al., 1990). Obstruction of lymphatics may result in lymphangioleiomyomas, chylothorax, and chylous ascites. Excessive proteolytic activity, which relates to an imbalance of the elastase/alpha1-antitrypsin system or metalloprotease (MMPs) and their inhibitors (tissue inhibitors of metalloproteases [TIMPs]) (Kitaichi et al., 1995), may be important in lung destruction and formation of cysts.Animal models suggest that estrogen may promote the metastasis of TSC2-deficient cells to the lungs.

Although primarily a disease of women of childbearing age, lymphangioleio myomatosis (LAM) has also been reported in postmenopausal patient (Silverstein et al., 1974). In this case the patient is 55 year old post menopausal.

Common lymphangioleiomyomatosis (LAM) symptoms include the following: Dyspnea, Cough.

Exacerbations of LAM have been reported to occur during pregnancy and menstruation, as well as with exogenous estrogen use.

LAM may be present in approximately 30$40 \%$ of all people with tuberous sclerosis complex (TSC), and up to $80 \%$ of older persons. In this case the patient presented with cough, shortness of breath no symptoms suggestive of pleural effusion or pneumothorax (Wahedna et al., 1994). 
The examination in lymphangioleiomyomatosis (LAM) is often normal. Signs of tuberous sclerosis complex may be present, and include the following:

- Facial angiofibromas

- Periungual fibromas

- Hypomelanotic macules, ash-leaf spots

The etiology of lymphangioleiomyomatosis (LAM) is unknown; however, the fact that the condition occurs primarily in premenopausal women and that it is exacerbated by high estrogen states suggests a role for hormones (Shen et al., 1987).

The link with TSC suggests a genetic component Lymphangioleiomyomatosis (LAM) complications include the following:

Pneumothorax; Hemoptysis; Osteoporosis osteoporosis is noted in this case.

\section{Differential Diagnosis}

Associations between lymphangioleiomyomatosis (LAM) and tuberous sclerosis complex (TSC) are as follows:

TSC is a predisposing condition

Several shared clinical features (eg, cystic lung disease.

Lymphatic disorders are as follows:

-Diffuse pulmonary lymphangiomatosis

-Lymphangiomas

-Pulmonary lymphangiectasis

Conditions of abnormal smooth muscle proliferation in the lung are as follows:

-Leiomyosarcoma

-Smooth-muscle proliferation in the lung -Benign metastasizing leiomyoma

Fig.1 Chest radiograph with bilateral reticulonodular shadows

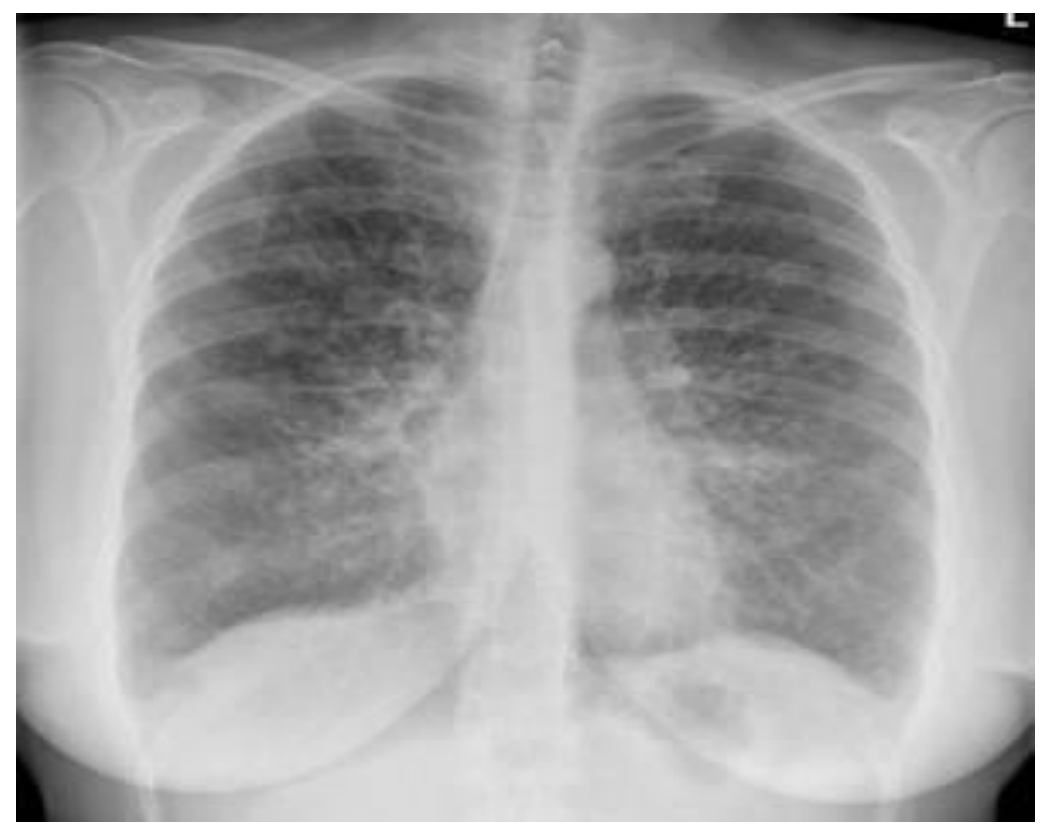


Fig.2 High resolution CT thorax showed multiple thin walled cysts scattered bilaterally with areas of interseptal thickening

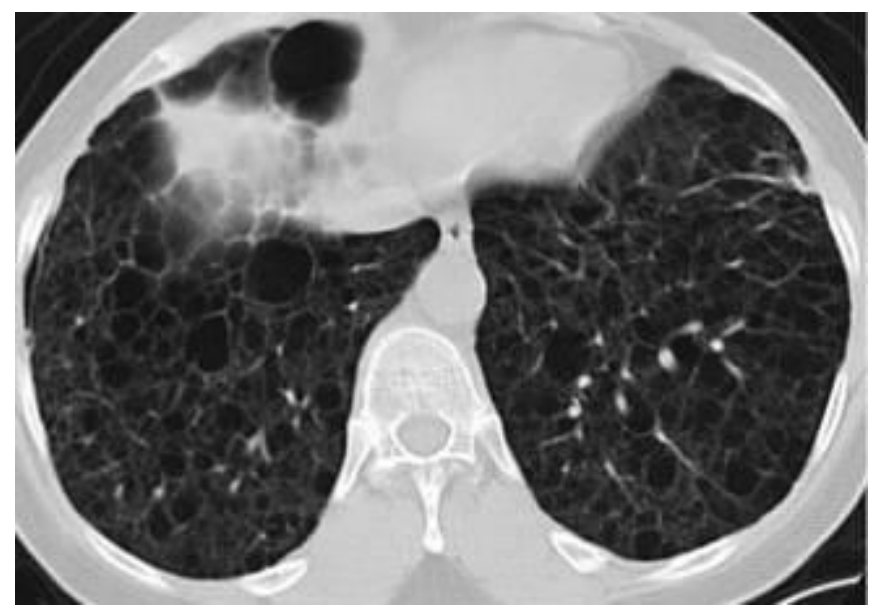

Fig.3 Photomicrograph of LAM lung tissue showing cystic structures and infiltration of the pulmonary parenchyma, airways, blood vessels, and lymphatics with smooth muscle cells.

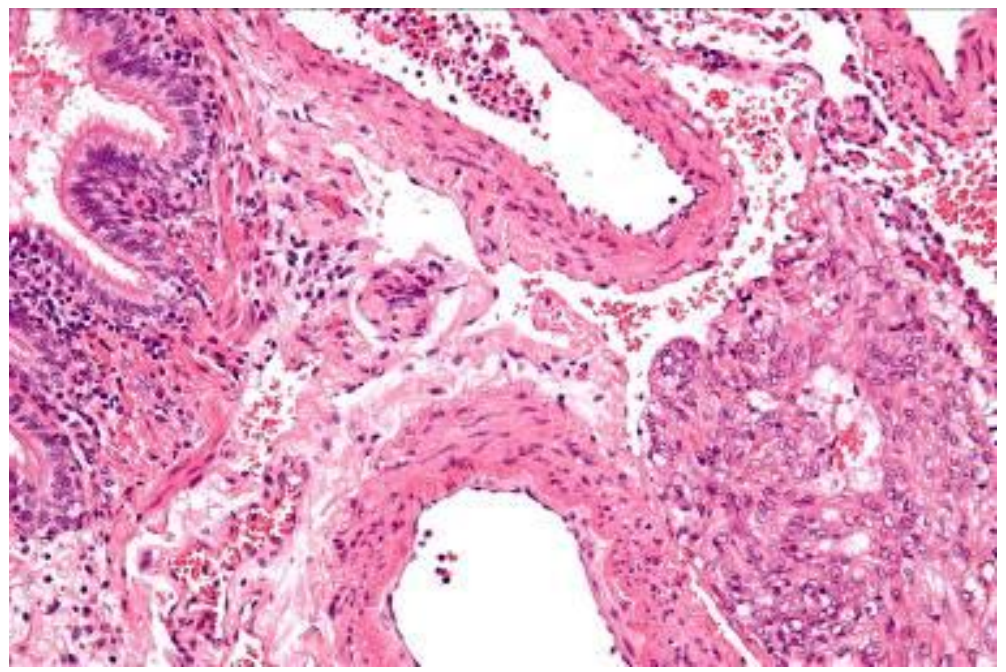

\section{Treatment}

\section{Sirolimus}

sirolimus (Rapamune) was the first drug approved by the US FDA for LAM. It has demonstrated improvement in lung function in patients with LAM. Sirolimus also reduces the size of angiomyolipomas, lymphangioleiomyomas, and chylous effusions. The therapeutic response,
However, is generally not sustained Rate of decline in lung function trends to be less in postmenopausal women (eg, surgical oophorectomy, age) we managed the case with inhaled corticosteroids and long acting $\mathrm{B}_{2}$ agonists and short course of antibiotics. patient showed significant improvement 
with improved Fev1. we dint use the sirolimus due to financial constrains. The subject was put on oral progesterone with $\mathrm{O}_{2}$ therapy. Patient showed good improvement in oxygen saturation and was discharged with oral progesterone and domiciliary oxygen supplementation. The case was reviewed after 6 months. There was significant improvement in dyspnea, spirometry, and arterial blood gas values. However, radiological features remained stationary with no deterioration

\section{References}

Brunelli, A., Catalini, G., Fianchini, A. 1996. Pregnancy exacerbating unsuspected mediastinal lymphangioleiomyomatosis and chylothorax. Int. J. Gynecol. Obstet., 52: 289-90.

Corrin, B., Liebow, A.A., Friedman, PJ.. Pulmonary lymphangioleiomyomatosis. Am. J. Pathol., 79: 34882.

Johnson, S. 1999. Rare disease. 1. Lymphangioleiomyomatosis: Clinical features, management and basic mechanisms. Thorax, 54: 254-64.

Kalassian, K.G., Raffin, T.A. 2000. Lymphangioleiomyomatosis. In: Murray JF, Nadel JA, editors. Text book of Respiratory Medicine. 3rd ed. Philadelphia: WB Saunders, p. 177588.

Kitaichi, M., Nishimura, K., Itoh, H., Izumi, T. 1995. Pulmonary lymphangioleiomyomatosis: A report of 46 patients including a clinicopathologic study of prognostic factors. Am. J. Respir. Crit. Care Med., 151: 527-33.

NHLBI. Workshop Summary. Report of workshop on lymphangioleiomyomatosis. National Heart, Lung, and Blood Institute. Am. J. Respir. Crit. Care Med., 159: 67983.

Shen, A., Iseman, M.D., Waldron, J.A., King, T.E. 1987. Exacerbation of pulmonary

lymphangioleiomyomatosis by exogenous estrogens. Chest, 91: 7825.

Silverstein, E.F., Ellis, K., Wolff, M., Jaretzki, A. 1974. 3rd. Pulmonary lymphangioleiomyomatosis. Am. J. Roentgenol Radium Ther. Nucl. Med., 120: 832-50.

Talmadge, E., King, Jr. 2008. Pulmonary lymphangioliomyomatosis. In:

Fishman's Pulmonary Diseases and Disorders. 4th ed. New York: Mac Graw Hill, p. 1255-63.

Taylor, J.R., Ryu, J., Colby, T.V., Raffin, T.A. $1990 . \quad$ Lymphangioleiomyomatosis. Clinical course in 32 patients. N. Engl. J. Med., 323: 125460.

Wahedna, I., Cooper, S., Williams, J., Paterson, I.C. 1994. Bri on JR, Ta ersfield AE, et al. Relation of pulmonary lymphangioleiomyomatosis to use of the oral contraceptive pill and fertility in the UK: A national case control study. Thorax, 49: 910-4.

\section{How to cite this article:}

Krishna Bharadwaj Reddy, V. Gopala Krishniaiah and Y. Murali. 2016. Lymphangioleiomyomatosis in a 55 Year Old Female -A Rare Case Report. Int.J.Curr.Microbiol.App.Sci. 5(7): 148-152. doi: http://dx.doi.org/10.20546/ijcmas.2016.507.014 